\title{
Hardware Implementation for the Echo Canceller System based Subband Technique using TMS320C6713 DSP Kit
}

\author{
Mahmod. A. Al Zubaidy \\ Ninevah University \\ Mosul, Iraq
}

\author{
Sura Z. Thanoon \\ (MSE student) School of Electronics Engineering \\ Mosul University \\ Mosul, Iraq
}

\begin{abstract}
The acoustic echo cancellation system is very important in the communication applications that are used these days; in view of this importance we have implemented this system practically by using DSP TMS320C6713 Starter Kit (DSK). The acoustic echo cancellation system was implemented based on 8 subbands techniques using Least Mean Square (LMS) algorithm and Normalized Least Mean Square (NLMS) algorithm. The system was evaluated by measuring the performance according to Echo Return Loss Enhancement (ERLE) factor and Mean Square Error (MSE) factor.
\end{abstract}

Keywords-Acoustic echo canceller; Least Mean Square (LMS); Normalized Least Mean Square (NLMS); TMS320C6713; 8 subbands adaptive filter

\section{INTRODUCTION}

The acoustic echo problem that appears in the communications systems was impeding the performance of systems efficiently and to solve this problem, it must define the echo and knowledge of its characteristics. Echo signal is defined as the delayed and attenuated version of the original signal produced by some device, such as a loud speaker. If we consider the transmitted signal $\mathrm{x}(\mathrm{t})$, then the attenuated and delayed version of it is the echo signal and it is given by the following (1) [1]:

$$
x d(t)=\alpha x(t-t d)
$$

Where $\alpha$ is the attenuation factor and td is time delay of the echo replica.

The echo signal starts to be sensible about the listener after 35 msecs and the amount of its delay and attenuation depend on the surface that the signal reflected from such as walls, floors and furniture, and the path that will travel through [2].

To solve this problem the adaptive filter was used to design acoustic echo cancellation system (AEC), where we will review the techniques used in the design of the adaptive filter in Section II, as well as the algorithms used in each technique in Sections III and IV. Then we present the circuits designed to delete the acoustic echo in Section V, performance evaluation in Section VI and the results obtained from each technique and algorithm used and the comparisons between the systems in Section VII followed with conclusions and future works in Sections VIII and IX, respectively.

\section{ADAPTIVE FILTER TECHNIQUES}

\section{A. Fullband Technique}

Fullband adaptive filter is the first solution to acoustic echo where in this way the whole sound would process by using one adaptive filter. Here the adaptive filter deals with the input that contains wide spectral dynamic range as one band so the speed of convergence would be slow. Fig. 1 shows Echo Canceller System using fullband adaptive filter method.

Fullband adaptive filter method suffers from two main problems which are [3]:

- First, when the input signal has wide spectral dynamic range such as that found in speech then the input correlation matrix is ill-conditioned or equivalently so that lead to be the convergence and tracking of a gradient-based adaptive filter can be very slow.

- Second, this method need very high-order adaptive filters and so it is computationally expensive. Subband adaptive filter technique used to overcome these problems, by decomposing the signal into subbands and each subband signal used separated adaptive filter.

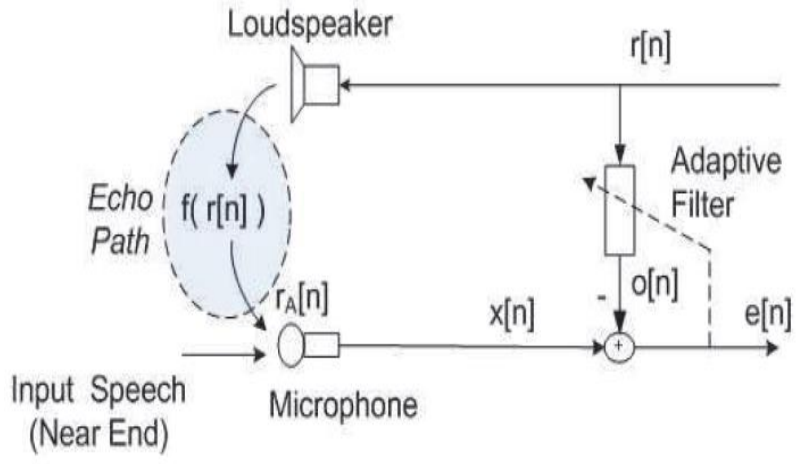

Fig. 1. Acoustic echo cancellation system.

\section{B. Subband Technique}

The subbands echo canceller technique consists of the subband analyzer which splits the input signal into $\mathrm{N}$ sub- 
bands, and $\mathrm{N}$ subband adaptive filter, each subband adaptive filter deals with one separated frequency band [4]. The main advantages of a subbands echo canceller are reduction in filter length, reduction in computational complexity and increasing the speed of convergence [2]. Fig. 2 shows the subband adaptive filter.

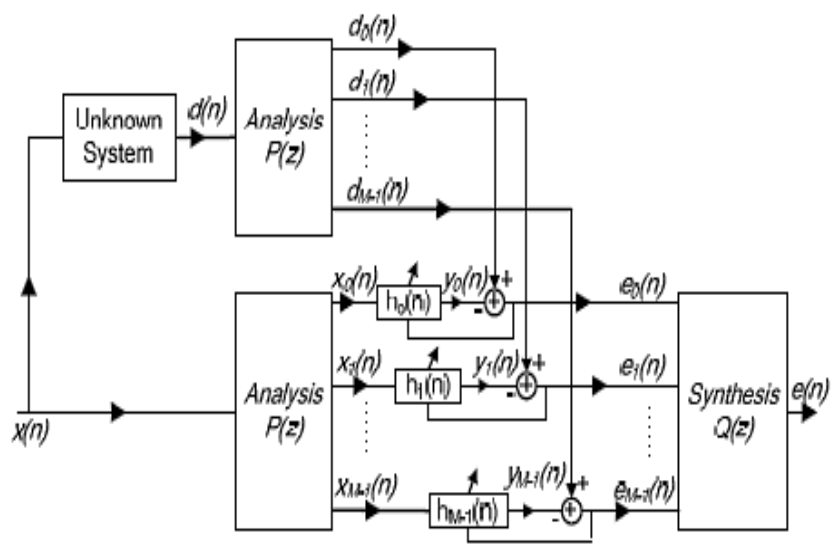

Fig. 2. Subband system identification.

However the adaptive filter can deal with any changing in the environments because it self-adjusting his weights continuously to get the highest performance for the system, the adaptive filter adjusts his weights according to algorithms such as LMS, NLMS, VLMS and RLMS. By choosing appropriate algorithm for the application, suitable length and step size for the filter, the performance of the designed system it becomes better.

\section{LEAST MEAN SQUARE ALGORITHM (LMS)}

The LMS adaptive filter is the first, most popular and widely used in adaptive system, appearing in numerous commercial and scientific applications the reasons behind this fame to it's easily in the implementation and there is no complexity in the calculation. The following equations described the LMS adaptive filter operation [5]:

$$
\begin{aligned}
& W(n+1)=W(n)+\mu(n) \text { e }(n) X(n) \\
& e(n)=d(n)-W^{T}(n) X(n)
\end{aligned}
$$

Where

$\mathrm{W}(\mathrm{n})=\left[\begin{array}{lll}\mathrm{w} 0(\mathrm{n}) & \mathrm{w} 1(\mathrm{n}) & \ldots \\ \mathrm{w} & \mathrm{wL}-1(\mathrm{n})\end{array}\right]^{\mathrm{T}}$ is the coefficient vector, $X(n)=[x(n) \quad x(n-1) x(n-L+1)]^{T}$ is the input signal vector, $d(n)$ is the desired signal, $e(n)$ is the error signal, and $\mu(\mathrm{n})$ is the step size.

\section{Normalized LEASt MEAN SQUARE Algorithm (NLMS)}

The NLMS adaptive filter is derivative form LMS adaptive filter where it is also easy to implement but with a change in the calculation of the step size where the step size is varying continuously to get a better performance where the convergence speed is increased and this property has been used in the design of acoustic echo cancellation system. The equation for calculating the step size is [6]:

$$
\mu=\frac{\beta}{c+\|x(n)\|^{2}}
$$

Where

$\mu(n)=$ step size, $\beta=$ Normalized step size $(0<\beta<2)$, c= small positive constant

The following equation to clarify how the filter calculates his weights [7]:

$$
\begin{aligned}
& \mathrm{w}(\mathrm{n}+1)=\mathrm{w}(\mathrm{n})+\mu(\mathrm{n}) \mathrm{e}(\mathrm{n}) \mathrm{x}(\mathrm{n}) \\
& \text { V. HARDWEAR IMPLEMENTATION FOR THE AEC SYSEM } \\
& \text { USING TMS320C6713 DSK }
\end{aligned}
$$

To implement AEC system in real-time required: one DSP TMS320C6713 Starter Kit (DSK), Two Personal Computers (PC) and Display Unit (Oscilloscope), first the system must design using MATLAB beside the Embedded Target for C6000 DSP, the procedure of the implementation in general was shown in Fig. 3.

The TMS320C6713 DSK starter kit is a low cost standalone DSP development platform that can be used to develop applications for the TMS320C67xx DSP family. It includes the C6713 floating-point digital signal processor (DSP) and a 32 bit stereo codec (AIC23) for input and output. The AIC23 codec uses a sigma delta technology that provides Analogue to Digital conversion (ADC) and Digital to Analogue conversion (DAC) and has got variable sampling rates from $8 \mathrm{kHz}$ to 96 $\mathrm{kHz}$. It includes $16 \mathrm{MB}$ synchronous dynamic random access memory (SDRAM) and $256 \mathrm{~KB}$ of flash memory. Furthermore it includes two inputs (LINE IN, MIC IN) and two output ports (LINE OUT, HEADPHONE). The DSK operates at a frequency of $225 \mathrm{MHz}$ and has got a single power supply of $5 \mathrm{~V}$ [8]. Fig. 4 and 5 show the photograph and the block diagram of the TMS320C6713 DSK KIT.

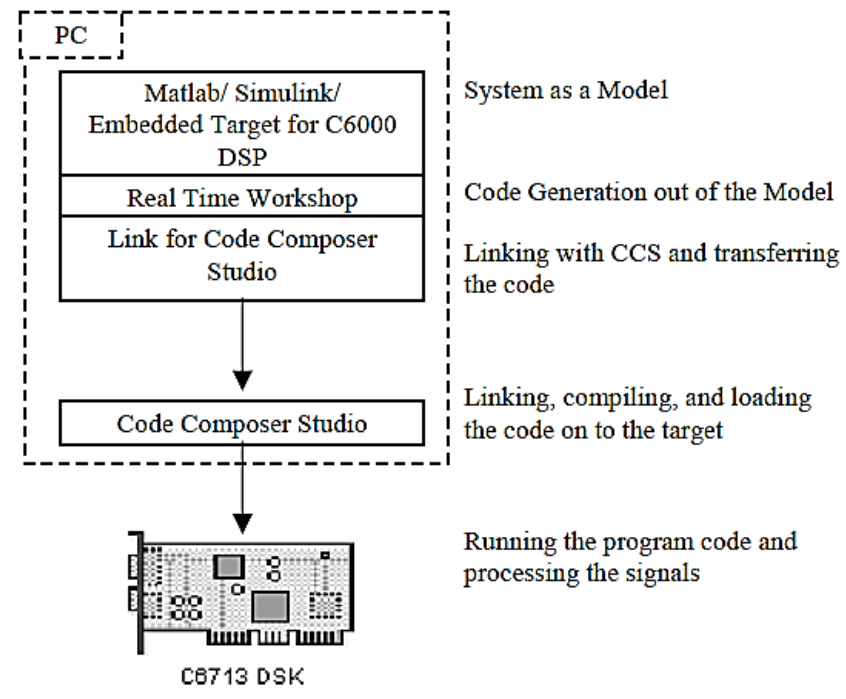

Fig. 3. Flow diagram of the procedures of implementation the model on C6713 DSK. 


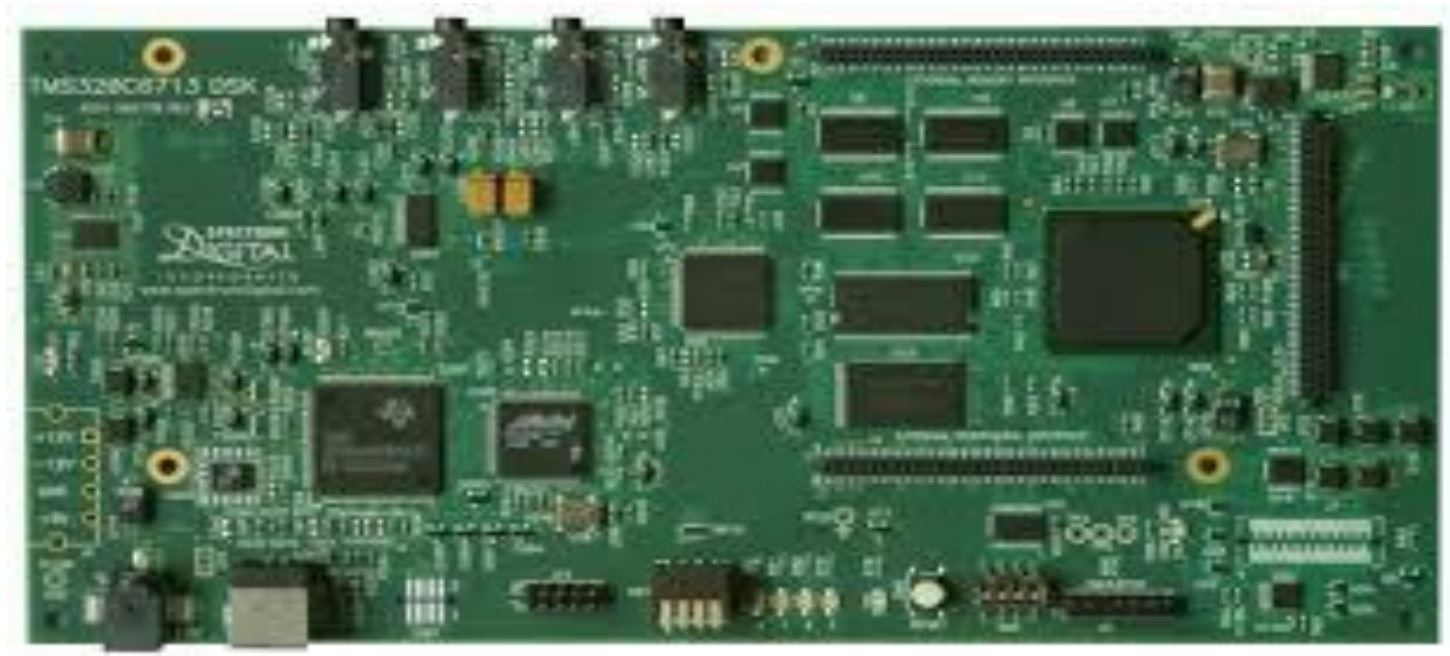

Fig. 4. The photograph of the C6713 DSK.

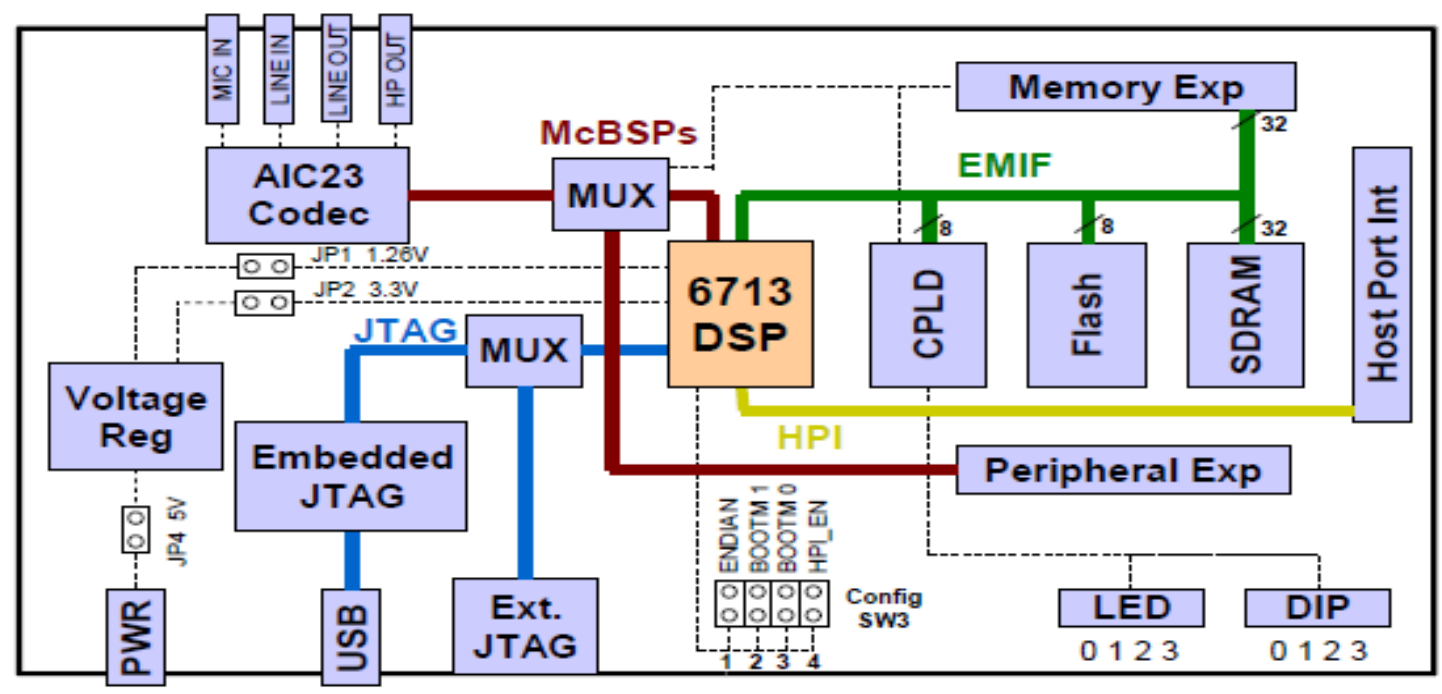

Fig. 5. C6713 DSK block diagram.

The real time hardware implementation of the AEC system needs two PCs, the first PC was employed to run the MATLAB model on DSP kit to install the definitions of the DSP kit. The second PC was used for running the models of sending the recorded speech to the DSP kit and saving the results, the two PCs must contain the MATLAB program.
By using MATLAB_SIMULINK program the fullband and 8 subband AEC system based LMS and NLMS algorithm for adaptive filter was designed and implemented on C6713 DSK, Fig. 6 shows the fullband AEC system based LMS and NLMS algorithm for the adaptive filter.

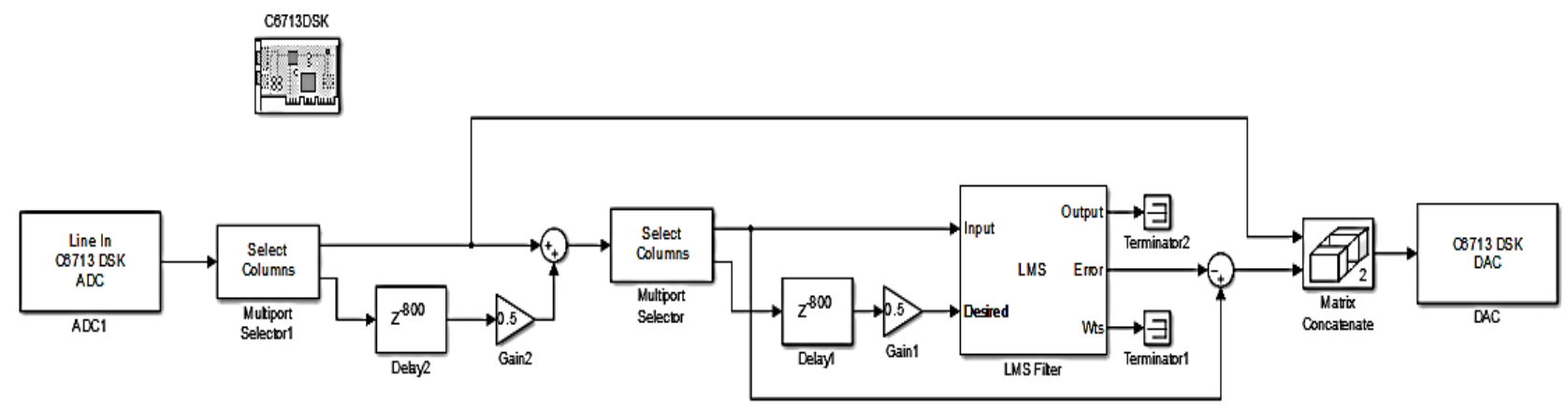

Fig. 6. Fullband AEC system using LMS algorithm and recorded speech signal as input on C6713 DSK. 


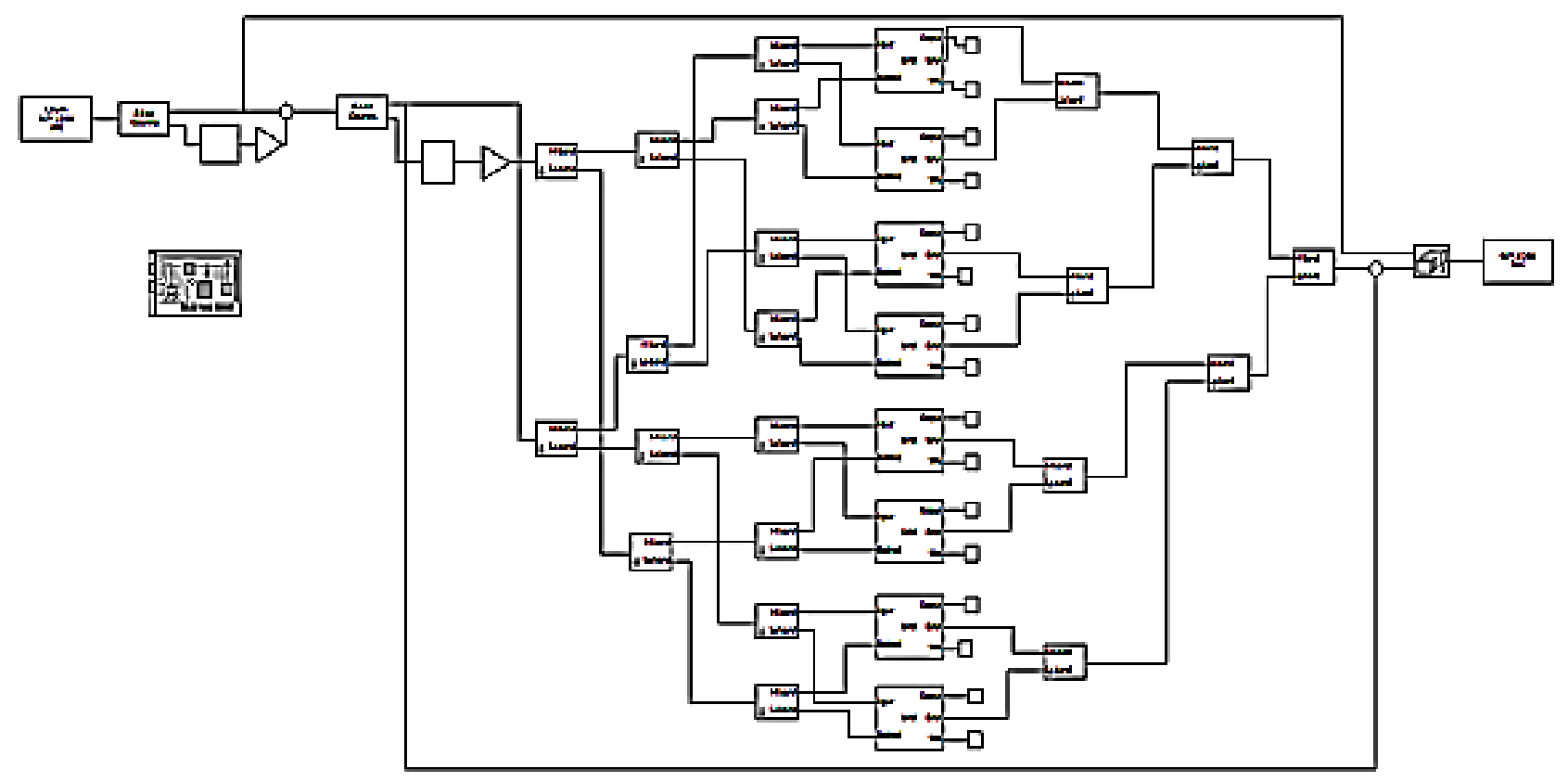

Fig. 7. 8 Subbands echo canceller system using LMS algorithm and recorded speech signal as input on C6713 DSK.

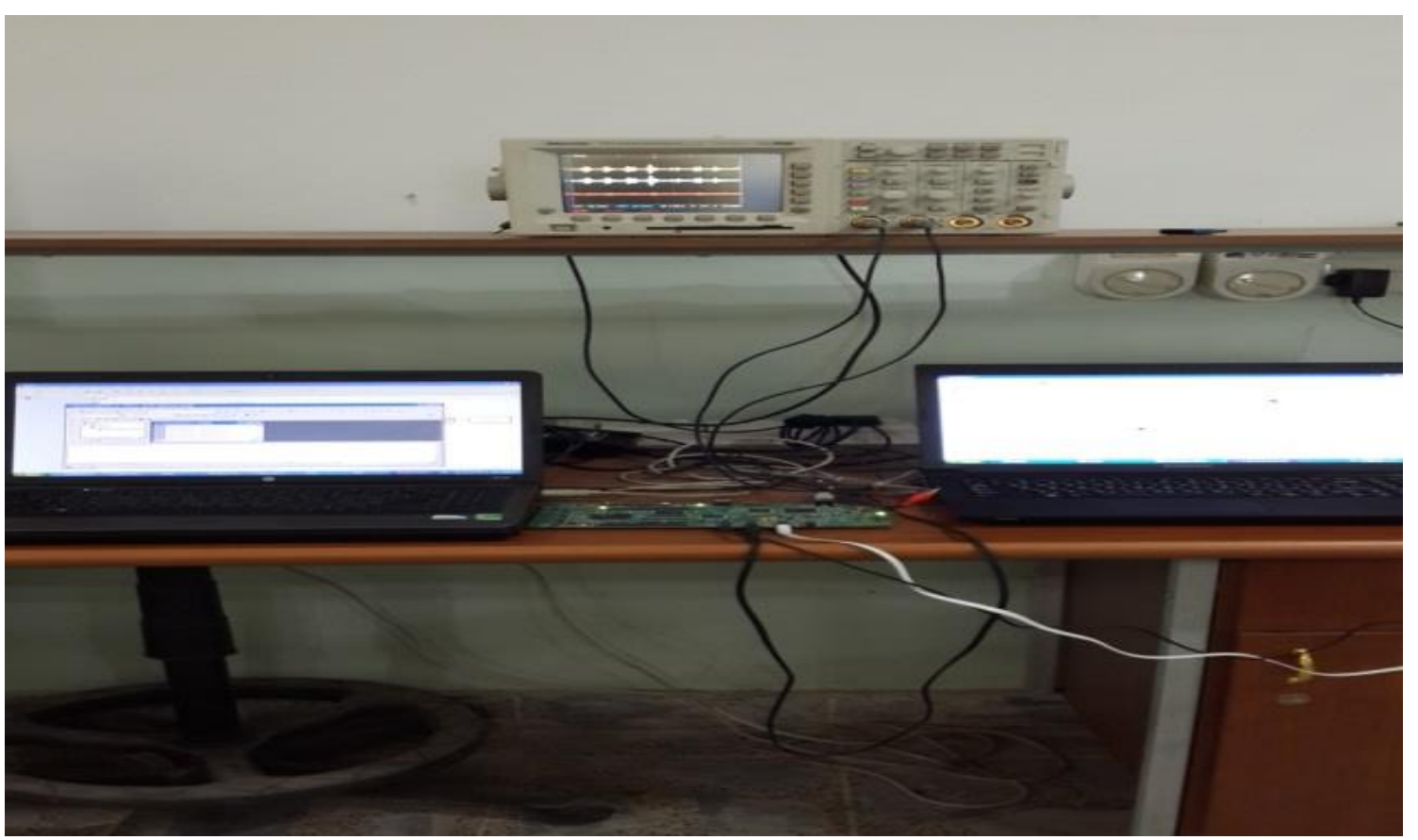

Fig. 8. The photograph of the hardware system implemented.

To display the results of the DSP kit a digital storage (500Msample/sec) oscilloscope was used to achieve this purpose. The complete hardware implemented system is shown in Fig. 8.

\section{Vi. Performance Evaluation}

The AEC system can be evaluated according to two factors, the echo return loss enhancement (ERLE) and mean square error (MSE). ERLE defined as the ratio of the is defined as the ratio of the microphone power signal, $d(n)$, to the power of the residual error signal after cancellation, e(n). ERLE measured in $\mathrm{dB}$ and it can be expressed as [9]:

$$
\operatorname{ERLE}=10 \log \frac{\mathrm{p}_{\mathrm{d}}(\mathrm{n})}{\mathrm{p}_{\mathrm{e}}(\mathrm{n})}=10 \log \frac{\mathrm{E}\left[\mathrm{d}^{2}(\mathrm{n})\right]}{\mathrm{E}\left[\mathrm{e}^{2}(\mathrm{n})\right]}
$$

The mean square error is measure give us the quantity of error level between two signals, or the similarity between them as shown in the following equation [10].

$$
\operatorname{MSE}=\frac{\sum \mathrm{e}^{2}}{\mathrm{n}}
$$




\section{HARDWEAR IMPLEMENTATION RESULTS}

The AEC system was implemented based TMS320C6713 DSK by using LMS and NLMS algorithm for the adaptive filter and choosing the parameters of the filter where the length of the filter was 1024 and the step size was 0.002 for both algorithms. The speech used to test the implemented system was recorded with sampling rate 8000 per second then the echo generated from this recorded speech with attenuation 0.5 and delay $100 \mathrm{msec}$ and summed with original speech to get the input signal to the system as shown in Fig. 7.

The output signals of the 8 subbands AEC system using LMS algorithm for the adaptive filter shown in Fig. 9.

The output signals of the 8 subbands AEC system using NLMS algorithm for the adaptive filter shown in Fig. 10.

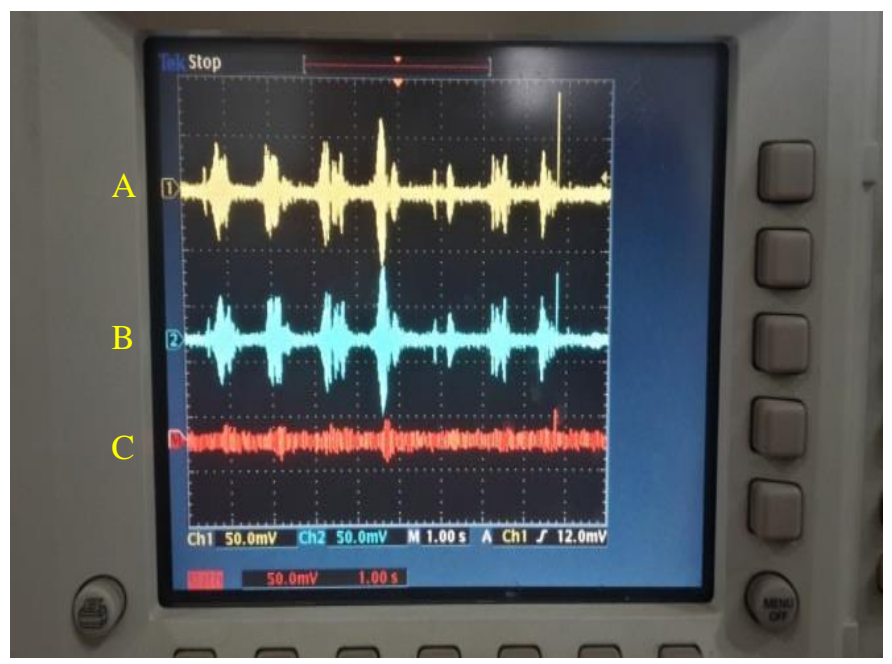

Fig. 9. 8 subbandsAEC system results using LMS algorithm and speech signal as input A- The original signal, B- The output signal, C- The error signal.

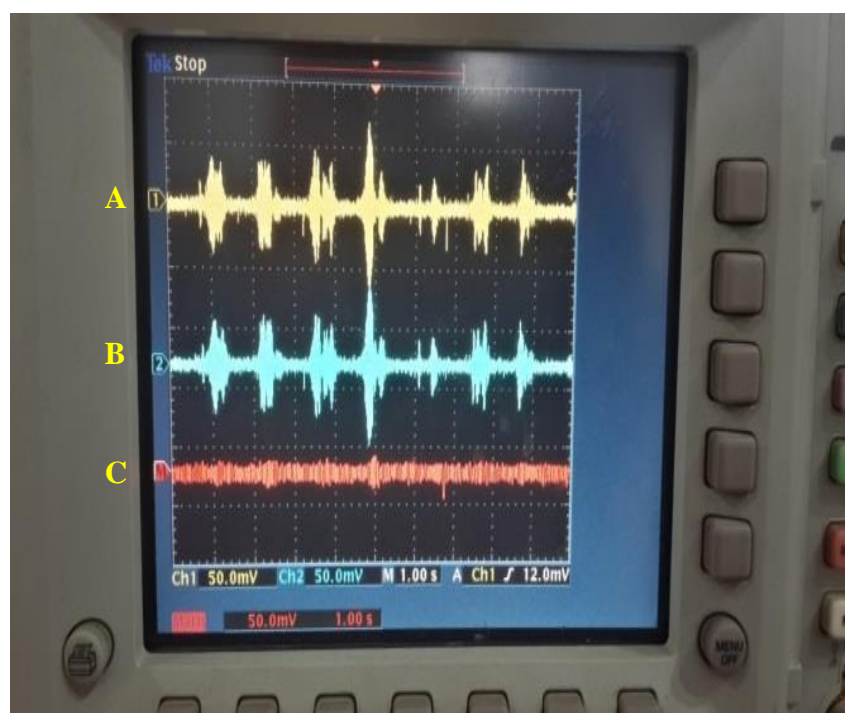

Fig. 10. 8 subbands AEC system results using NLMS algorithm and speech signal as input A- The original signal, B- the output signal, C- the error signal.
By comparing the output signals of the both systems it can be notice that the error signal in Fig. 10 is less than the error signal in Fig. 9, and by calculating ERLE and MSE factors for these two systems, For LMS algorithm ERLE was $11.23 \mathrm{~dB}$ and MSE was 0.0000408189 and for NLMS algorithm ERLE was $13.56 \mathrm{~dB}$ and MSE was 0.0000279061 .

By comparing between the above results for LMS algorithm and NLMS algorithm the different in the performance was clearly noticed and proof that NLMS algorithm is better than LMS algorithm as shown in Fig. 11 and 12 .

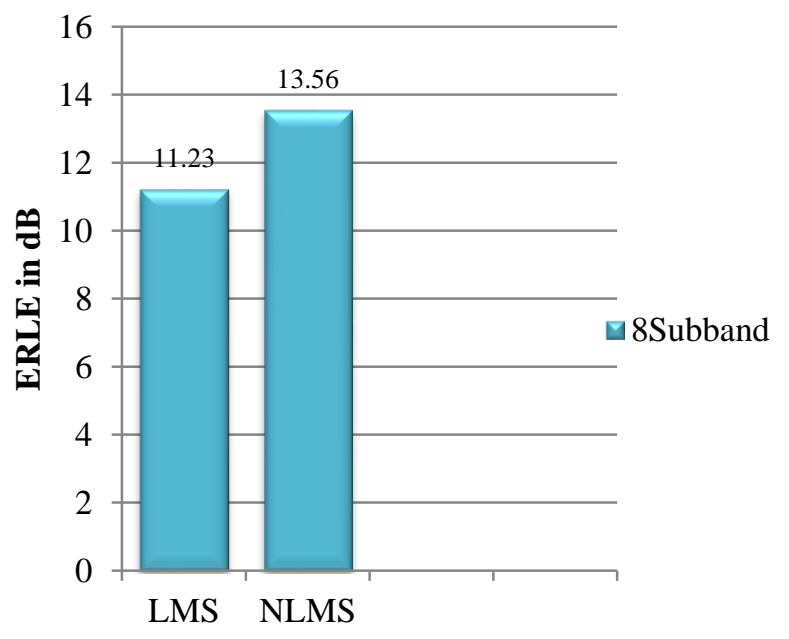

Fig. 11. ERLE values for the 8subbandsAEC system on C6713 DSK based on LMS and NLMS tested by speech input signal.

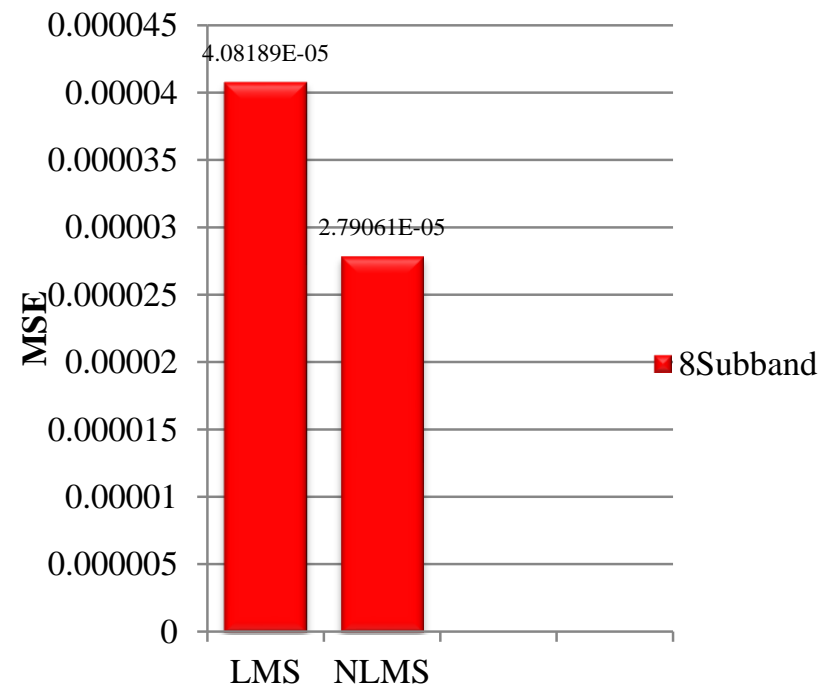

Fig. 12. MSE values for the 8subbandsAEC system on C6713 DSK based on LMS and NLMS tested by speech input signal. 


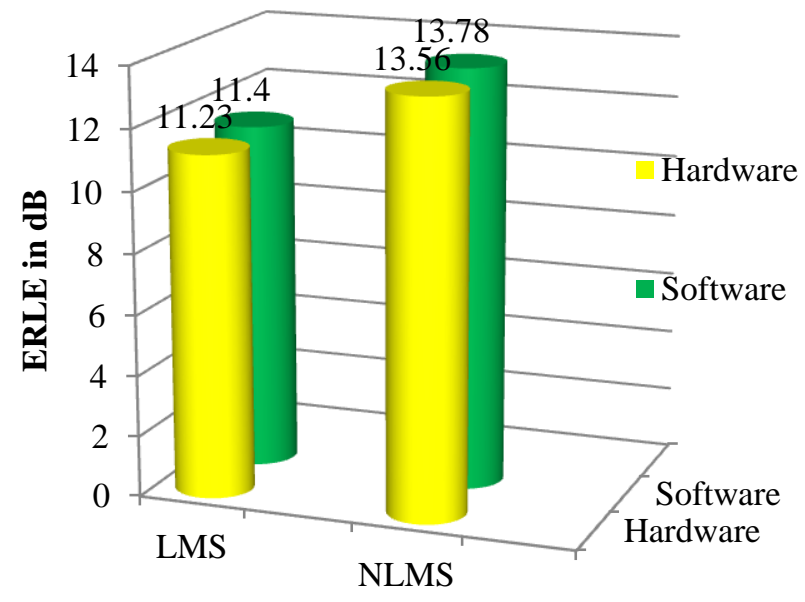

Fig. 13. Comparison between software and hardware results in ERLE values of the AEC system using LMS and NLMS algorithm and recorded speech as input signal.

The AEC system was designed primary by using MATLAB SIMULINK and tested also by using the same recorded speech, by looking at the results of the simulation model and compare it with the hardware results it can by notice that the results using TMS320C6713 DSK converge from the simulation results where the different is very small as shown in Fig. 13 [11].

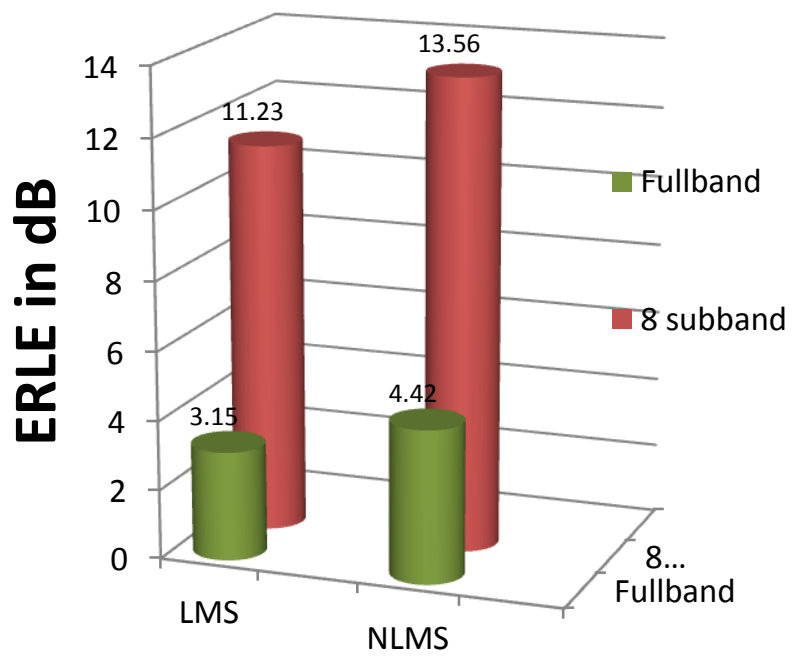

Fig. 14. Comparison between fullband and 8subband techniques in ERLE values of the AEC system using LMS and NLMS algorithm and recorded speech as input signal.

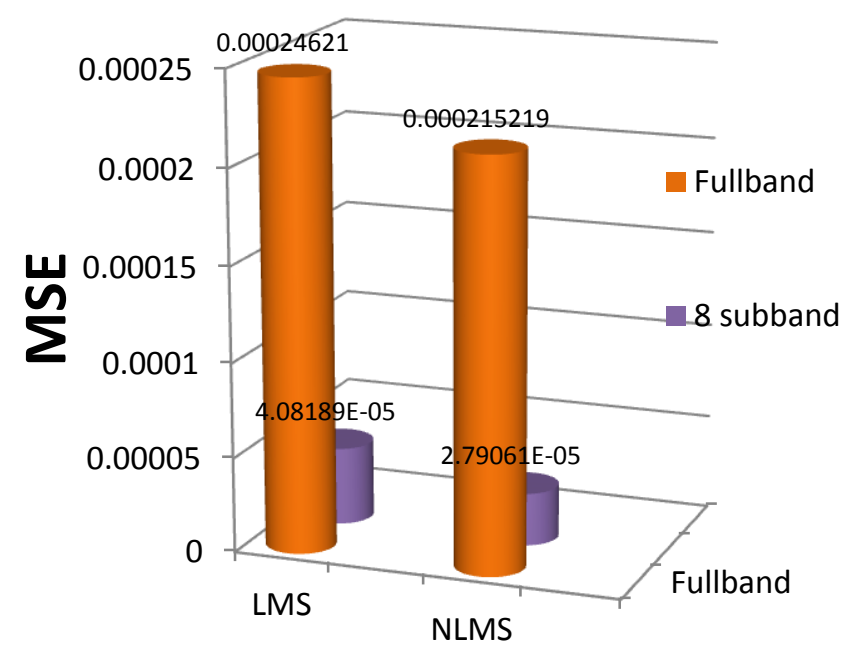

Fig. 15. Comparison between fullband and 8subband techniques in MSE values of the AEC system using LMS and NLMS algorithm and recorded speech as input signal.

The AEC system was implemented using two technique fullband and 8 subband, to determine which system cancel the echo signal more efficiently we compare the results between these two systems according to ERLE and MSE factors. In AEC system using fullband adaptive filter, for LMS algorithm, ERLE was $3.15 \mathrm{~dB}$, and MSE was 0.00024621. For NLMS algorithm, ERLE was $4.42 \mathrm{~dB}$, and MSE was 0.000215219 . Comparing the results using fullband technique with the results when using 8 subband technique either using LMS or NLMS algorithm it can be notice that 8 subband technique is better than fullband technique as Fig. 14 and 15 shown.

\section{CONCLUSION}

1) The Echo Cancellation system is an effective system for deleting acoustic echo by generating an echo similar to the echo comes with the original signal using an adaptive filter and then subtracting it from the received signal.

2) The appropriate option for implementing the systems in the real-timer is to use TMS320C6713 DSP kit.

3) Returning to the results obtained from the hardware and using the factors of evaluation ERLE and MSE we find that the NLMS algorithm that used for the adaptive filter is better than the LMS algorithm.

\section{FUTURE WORK}

1) Using 16 subbands technique to implement $\mathrm{AEC}$ system.

2) Implementation of AEC system in mobile applications. 


\section{REFERENCES}

[1] Artur Ferreira and Paulo Marques, (2011), "Echo Cancellation for Hands-Free Systems", Adaptive Filtering, DrLino Garcia (Ed.), ISBN: 978-953-307-158-9, InTech, Available from: http://www.intechopen.com/books/adaptive-filtering/echo cancellation for-hands-free-systems.

[2] Saeed V. Vaseghi, "Advanced Digital Signal Processing and Noise Reduction", Second Edition, ISBNS: 0-471-62692-9 (Hardback): 0-47084162-1 (Electronic), 2000.

[3] Chaitanya M. Patil and R.A.Deshpande, "Comparative Analysis Based on Statistical Parameters of LMS and NLMS Algorithms on ECG Signal by Using Matlab Simulink", International Journal of Advances in Electronics and Computer Science, Vol. 2, Issue 8, August 2015.

[4] Phillip L. De Le_on II and Delores M. Etter, "Acoustic echo cancellation using subband adaptive filtering ", ch 11 wavelet book.pdf

[5] Widrow, B. and Hoff, M.E., "Adaptive switching circuits", IRE WESCON Conv. Rec.,4, 96-104, Aug. 1960. Tavel, P. 2007 Modeling and Simulation Design. AK Peters Ltd.
[6] Monsoon H. Hayes, "Statistical Digital Signal Processing and Modeling", John Wiley \& Sons, Inc., 1992.

[7] J. Dhiman, S. Ahmad and K. Gulia, "Comparison between adaptivefilter algorithms (lms, nlms and rls)", International Journal of Science,Engineering and Technology Research, vol. 2, no. 5, pp. pp1100, 2013.

[8] E Kaymak , M A Atherton, K R G Rotter and B Millar, " Real-time adaptive filtering of dental drill noise using a digital signal processor", Conference Paper, June, 2006.

[9] SrinivasaprasathRaghavendran, "Implementation of an acoustic echo canceller using MATLAB", University of South Florida, 2003.

[10] Zhou Wang and Alan C. Bovik, "Mean Squared Error: Love It or Leave It?",IEEE,pp 98-117,JANUARY 2009.

[11] Mahmod. A. Al Zubaidy and Sura. Z. Thanoon, "Evaluation and the Performance of LMS and NLMS Algorithm in Acoustic Echo Cancellation using the 8 sub-bands Techniques", International Journal of Engineering and Innovative Technology (IJEIT), Volume 7, Issue 3, PP. 1-3, September 2017. 